\title{
Déjà vu experiences in anxiety
}

Christine E. Wells ${ }^{a *}$, Akira R. O’Connor ${ }^{\mathrm{b}}$ and Chris J.A. Moulin ${ }^{\mathrm{c}}$

${ }^{a}$ School of Social and Health Sciences, Leeds Trinity University, Leeds, UK; ${ }^{b}$ School of Psychology and Neuroscience, University of St Andrews, St Andrews, UK, Email: aro2@st-andrews.ac.uk; Laboratoire de Psychologie et Neurocognition, LPNC CNRS 5105, Université Grenoble Alpes, France, Email: chris.moulin@me.com

*Email: ch.wells@leedstrinity.ac.uk; Address: School of Social and Health Sciences, Leeds Trinity University, Brownberrie Lane, Horsforth, Leeds, LS18 5HD 


\section{Déjà vu experiences in anxiety}

Déjà vu occurs when a novel event is experienced with an erroneous sense of familiarity. Memory researchers theorise that this arises due to an error in the processes underlying the recognition memory system. Research has indicated that there may be a link between high levels of anxiety and increased frequency and intensity of déjà vu, however there has been comparatively little characterisation of déjà vu as experienced by individuals with clinical anxiety. We used an online questionnaire to collect data from individuals self-reporting a clinical diagnosis of anxiety, as well as from age-matched controls. The Anxiety Group reported a significantly higher frequency of déjà vu episodes over the previous month than controls. They also reported experiencing déjà vu more frequently and with higher intensity during periods of high anxiety. In addition, the Anxiety Group reported finding déjà vu episodes significantly more distressing than the control group. The findings indicate that there are differences in déjà vu experienced by people reporting high levels of anxiety compared to healthy controls without an anxiety diagnosis. We discuss structural and neural mechanisms thought to underpin déjà vu in relation to these results.

Keywords: déjà vu; anxiety; memory; decoupled familiarity; theta

\section{Introduction}

Déjà vu is a phenomenon conservatively thought to be experienced by approximately $60 \%$ of the general population (Brown, 2003). It is typically a transitory state during which a person encountering a novel experience, such as visiting a new place, is struck by an erroneous sense of familiarity (Illman, Butler, Souchay \& Moulin, 2012). Crucially, the déjà vu experience is accompanied by insight into the false nature of this feeling of familiarity (e.g. Urquhart, Sivakumaran, Macfarlane \& O’Connor, 2018).

Whilst the mechanisms underpinning déjà vu are yet to be precisely defined, researchers have identified several potential neural correlates. Brázdil et al (2012) used source-based morphometry to compare the MRI scans of healthy participants who either 
had or had not experienced déjà vu. Group allocation was determined by their responses on the Inventory for déjà vu experiences assessment (IDEA; Sno et al, 1994), a retrospective measure of déjà vu experiences. There was significantly less grey matter in several regions of the limbic-temporal network including the hippocampi and parahippocampal gyri, insular cortices, superior temporal sulci, basal ganglia and thalami in participants who had experienced déjà vu. In addition,grey matter volume in these regions correlated inversely with déjà vu frequency. These volumetric differences are consistent with grey matter reductions reported in patients with temporal lobe epilepsy (TLE, e.g. Brázdil, Mareček, Fojtíková et al, 2009; Pail, Brázdil, Mareček\& Mikl, 2010). TLE research has provided insight into the mechanisms underlying déjà $\mathrm{vu}$ as these experiences often feature as part of simple partial seizures. Similarities have been noted between TLE-related and 'healthy' (i.e. non-pathological) déjà vu (WarrenGash \& Zeman, 2003; Moulin 2014) in terms of the phenomenology and intensity of these episodes, although it may be that déjà vu symptoms are on a continuum between healthy and epileptic déjà vu (Perucca et al, 2017). Labate et al (2015) compared healthy participants who had and had not experienced déjà vu and patients with benign TLE who did or did not experience déjà vu as part of their epilepsy. The TLE group with déjà vu had increased grey matter volume in the left mesio-temporal region and left visual cortex compared with TLE patients without déjà vu. The healthy déjà vu group demonstrated a different profile, principally in terms of reduced grey matter volume in the anterior insular cortex, part of the limbic system, compared to the healthy non-déjà vu group. The authors summarise that alterations in memory circuitry are present in individuals with epilepsy and déjà vu, whilst alterations are seen in emotional circuitry in healthy individuals experiencing déjà vu. 
Whilst these studies implicate regions, they do not identify whether déjà vu is a result of the functional interactions between this set of regions. Shaw, Marecek \& Brázdil, 2016) examined structural covariance, an indicator of the organisation of neural signalling between constituent brain structures, in healthy déjà vu experiencers. They reported that pairwise correlations in grey matter amongst regions within the medial temporal lobe (MTL) and between MTL, dorsal striatum and insula cortex were more positively correlated as self-reported frequency of déjà vu episodes increased. As such, the authors proposed that healthy déjà vu results from some form of aberrant signalling within MTL circuitry.

TLE patients who have undergone electrical stimulation of MTL regions and related structures using depth electrodes have also provided useful insights into the underlying circuitry of déjà vu. Stimulation of the rhinal cortex has been shown to induce déjà vu episodes (e.g. Bancaud, Brunet-Bourgin, Chavel \& Halgren, 1994; Barbeau et al, 2005; Bartolomei et al, 2012). In addition, rhinal stimulation has indicated that theta band activity, involved in memory processing, may be linked to déjà vu episodes, as stimulation-induced episodes have been observed to be accompanied by theta synchronisation across MTL structures (Barbeau et al; Bartolomei et al, 2012).

The implication of the MTL and associated structures in déjà vu fits well with the models of déjà vu implicating recollection and familiarity (Brown \& Aggleton, 2001; Eichenbaum, 2007; Squire et al, 2007). For example, according to the decoupled familiarity hypothesis (Illman et al, 2012), déjà vu can be conceptualised as a shortlived neurological event which leads to a disruption of recognition memory, in which the subjective feelings associated with memory retrieval become dissociated from the actual memory retrieval. Specific to déjà vécu, a clinical manifestation of déjà vu, O’Connor, Lever \& Moulin (2010) speculatively proposed a mechanism by which 
systematically mistimed neural signalling could result in erroneous recollection of novel stimuli. Briefly, they proposed that hippocampal CA1 neuron firing, decoupled from the usual point on the cycle indicating novelty, and mistimed to the point that it systematically occurs at a phase of the theta cycle consistent with recollection, would result in behaviours seen in patients experiencing pathological déjà vu characterised by accompanying confabulation.

The putative relationship with the theta cycle provides a link between déjà vu and associated emotions. The theta cycle is implicated in memory-related processes (e.g. Mormann et al, 2008; Osipova et al, 2006; Sederberg, Kahana, Howard, Donner \& Madsden, 2003) but also in emotional processing, specifically anxiety (Gray, 1982; Gray \& McNaughton (2000). Rodent hippocampal theta is reliably reduced through the administration of a range of neurochemically dissimilar anxiolytic drugs (Gray \& McNaughton, 2000; Wells et al, 2013). Crucially, this effect is not observed with other types of psychoactive drugs e.g. antipsychotics, leading to the suggestion that this a reliable index of a reduction in anxiety. There is also evidence in the animal literature to suggest increased anxiety is associated with alterations in theta (e.g. Fontani, Farabollini \& Carli, 1984; Fontani \& Vengi, 1990a \& b; Gordon, Lacefield, Kentros \& Hen, 2005; Meyza, Boguszewski, Olszewski, Kasicki \& Zagrodza, 2009). Human data also supports the role of theta oscillations in anxiety (e.g. Cornwell, Arkin, Overstreet, Carver, \& Grillon, 2012; Khemka, Barnes, Dolan \& Bach, 2017).

Whilst the déjà vu literature has placed considerable focus on TLE, there is a small amount of literature which suggests déjà vu experiences differ in individuals with anxiety compared to those with TLE. Harper and Roth (1962) conducted structured interviews with 30 individuals with what they termed 'phobic-anxiety-depersonalisation syndrome' (PADS; Roth, 1959), a diagnosis characterised by both agoraphobic - like 
symptoms and depersonalisation, and 30 individuals with TLE, aiming to compare the prevalence of a range of symptoms. Déjà vu was reported by 12/30 of the PADS and 7/30 of the TLE group. The authors also indicated that déjà vu episodes were of a longer duration in the PADS group (although this data was not presented in the article). These findings therefore indicate a possible link between symptoms associated with anxiety and a higher frequency of occurrence and longer duration of déjà vu episodes.

Interest in exploring the relationship between anxiety and déjà vu was recently revived through a case study reported by Wells et al. (2014). A young male, presenting with high levels of anxiety, reported experiencing chronic and debilitating déjà vu. Neurological assessment indicated no obvious underling neurological pathology, indicating that this was potentially psychogenic in nature, and that his high level of anxiety may both contribute to and result from his frequent déjà vu experiences. It is difficult to characterise the nature of the relationship between anxiety and déjà vu from this single case but, placed alongside the earlier findings of Harper and Roth (1962), it adds to the rationale behind conducting a more extensive assessment of the déjà vu experiences of clinically-anxious patients compared to healthy controls and to further define the nature of the relationship between anxiety and déjà vu.

There is also a broader rationale for exploring the relationship between anxiety and déjà vu. There has been acknowledgement within the literature that déjà vu can be symptomatic of psychiatric disorders (Warren-Gash \& Zeman, 2003), yet beyond a few studies which have explored déjà vu in the context of schizophrenia (Adachi et al, 1999) and mood disorders (Silberman, Post, Nurnberger, Theodore \& Boulenger, 1985), there is limited data characterising déjà vu in clinical groups besides TLE. Therefore, there remains both scope and rationale to profile déjà vu in a range of psychiatric disorders in considerably more detail than currently exists, and anxiety disorders have been 
highlighted as of particular interest (Illman et al, 2012; Moulin, 2014; Wells et al, 2014).

This paper presents the results of a questionnaire study assessing aspects of déjà $\mathrm{vu}$, including frequency, intensity, and triggers of these episodes in two groups of participants: individuals who self-reported having a clinical diagnosis of anxiety ('Anxiety Group') and individuals who reported having no such diagnosis ('Control Group') and compared responses across these two groups. The study also included several standardised measures used to assess anxiety, stress, depression and dissociation. Based on the nascent literature in the field, we speculatively suggest that clinically anxious participants will report their déjà vu experiences as being more frequent and longer in duration than non-anxious controls.

\section{Method}

\section{Participants}

All participants were recruited online from advertisements posted on a range of sites, including anxiety websites and forums, social media and websites recruiting voluntary psychology research participants. Participants were given the choice to complete the study online or request paper copies. The majority of participants $(99.4 \%)$ completed the study online. Two participants requested paper copies, which were posted to them for completion.

\section{Demographic characteristics}

A total of 352 participants completed the study. Of these, 153 participants reported having a diagnosis of clinical anxiety ( 23 males, 130 females, mean age $=26.8$ years, $\mathrm{SD}=9.1$ years), described as the clinical anxiety group. The participants with 
clinical anxiety were also asked to provide their anxiety diagnosis from several alternatives taken from the DSM-IV criteria. The most frequently reported diagnosis was Generalised Anxiety Disorder (GAD; $63.9 \%$ of participants). The anxiety group also reported diagnoses of Social Phobia (28.8\%), Post-Traumatic Stress Disorder (PTSD; 19.6\%), Obsessive Compulsive Disorder (OCD; 13.7\%), Specific Phobia (4.6\%), Acute Stress disorder (1.3\%), Panic Disorder with Agoraphobia (9.8\%), without Agoraphobia (12.4\%), Agoraphobia without history of Panic Disorder (2.6\%). Sixtyfive $(42.5 \%)$ of the Anxiety Group reported comorbid anxiety diagnoses. There were 199 participants in the healthy control group (41 males, 156 females, 2 did not report gender, mean age $=25.4, \mathrm{SD}=8.0$ years).

The mean age of the Anxiety group (26.78 $[\mathrm{SD}=9.11$, range $=18-66]$ years $)$ was comparable to the control group $\left(25.49[\mathrm{SD}=8.04\right.$, range $=19-61]$ years; $\mathrm{t}_{350}=$ $1.42, \mathrm{p}=0.16, d=0.15)$. There were $23(15.0 \%)$ males and $130(85.0 \%)$ females in the Anxiety group. The control group comprised 41 (20.6\%) males, 156 (78.4\%) females and $2(1.0 \%)$ participants didn't provide gender information.

A series of t-tests on our standardised measures (see below) ascertained that our Anxiety Group were significantly more anxious than our control group (see Table 1). In sum, the Anxiety group scored significantly higher on all measures assessing anxiety, stress, depression and dissociation.

[Table 1 here]

\section{Measures}

The questionnaire study comprised five sub-sections 1) Demographics; 2) Questions about anxiety and any clinical anxiety diagnoses (N.B. this section included the following question: 'On a scale of $1-5$, how would you rate your overall anxiety 
levels compared to other people?' - this is referred to as 'subjective anxiety' throughout the rest of the paper); 3) An assessment of general knowledge about déjà vu and any such experiences participants have had to date; 4) Several standardised measures to assess levels of anxiety, depression, stress, and dissociative experiences; and 5) Questions asking more specifically about participants' déjà vu in relation to anxiety. Figure 1 presents an overview of the structure of the questionnaire and déjà vu questions. The questionnaire battery was identical in terms of content and ordering of the questions in both the online and paper versions.

\section{Déjà vu assessment}

Before answering questions about déjà vu, participants were provided with a definition: 'Déjà vu is the name we give to a strange sensation, where we feel like we have encountered something before, but we know that we have not. It might be meeting a person for the first time, going to a new place, or just having a conversation'. They were then asked two sets of questions about déjà vu. These were positioned either side of the standardised measures of anxiety, depression, stress and dissociative experiences. Part 1 asked participants about their previous experiences of déjà vu (e.g. frequency and duration of déjà vu experiences, Figure 1). Part 2 included questions which focused more specifically on déjà vu in the context of anxiety (e.g. frequency of déjà vu experiences, Figure 1). This section also included a number of open-ended questions about déjà vu, which asked participants to elaborate on issues including: whether they ever experienced any abnormal sensations during their déjà vu experiences; whether their déjà vu experiences contained any emotional content; whether they had ever noted a connection between their déjà vu experiences and anxiety or discussed this relationship with anyone. They were also asked whether they had ever discussed their déjà vu experiences with a professional and what they perceived their attitude to be. A 
limited number of responses were gathered for these open-ended questions and so the analysis presented here is primarily focused on the quantitative data gathered from the questionnaire.

Standardised measures of dissociation, anxiety, stress and depression

\section{Dissociative Experiences Scale}

The Dissociative Experiences Scale (DES; Bernstein \& Putnam, 1993) is a 28 item self-report questionnaire assessing dissociation. An example item is 'Some people have the experience of finding themselves in a place and having no idea how they got there. Select a number to show what percentage of the time this happens to you.' Participants select a percentage value ranging from $0-100 \%$ in $10 \%$ intervals. Higher mean scores reflect higher levels of dissociation.

\section{Depression, Anxiety and Stress Scales}

The Depression, Anxiety and Stress Scales (DASS-21) measure the dimensions of depression, anxiety and stress (Lovibond \& Lovibond, 1995). The 21-item version includes 7 statements for each of the three dimensions. Participants are asked to indicate how much each statement applied to them over the past week. An example item from the anxiety scale is 'I worried about situations in which I might panic and make a fool of myself'. Participants are asked to rate each item from on a 4-point Likert scale from 'Did not apply to me at all' to 'Applied to me very much, or most of the time'. The total score is multiplied by 2 to calculate the final score. Scores for each dimension can fall into one of 5 categories; normal, mild, moderate, severe and extremely severe (Depression: normal 0-9; mild 10-13; moderate 14-20; severe 21-27; extremely severe 28+; Anxiety: normal 0-7; mild 8-9; moderate 10-14; severe 15-19; extremely severe 
20+; Stress: normal 0-14; mild 15-18; moderate 19-25; severe 26-33; extremely severe $34+)$.

Zung Self-Rating Anxiety Scale (SAS)

The Zung Self-Rating Anxiety Scale (Zung, 1971), is a 20-item questionnaire assessing cognitive and somatic symptoms of anxiety. Participants are asked to indicate how much each statement applied to them over the past week. An example item from the scale is 'I get upset easily or feel panicky'. Rating is on a 4-point Likert scale from 'Never/rarely' to 'Always'. Scoring involves the reversal of five items, and total scores are then converted to anxiety index scores. Scores below 45 are considered within the normal range, 45-59 = minimal to moderate anxiety, 60-74 = marked to severe anxiety, $\geq 75$ most extreme anxiety.

\section{Major Depression Inventory (MDI)}

The Major Depression Inventory (MDI; Bech, Rasmussen, Olsen, Noerholm \& Abilgaard, 2001) consists of 10 items which cover the ICD 10 and DSM-IV symptoms of Major Depression. Items 8 and 10 are divided into two sub-items, and the highest scoring of each of these is included in the total score. Participants are asked to indicate how they have been feeling over the past two weeks. An example item is 'Have you felt in low spirits or sad?' Rating is on a 6-point Likert scale from 0 (symptom is never present) to 5 (symptom constantly present). Scores of 20-24 reflect mild depression, 2529 moderate depression, and 30+ are considered to reflect severe depression.

\section{Procedure}

Participants were recruited online from a range of sources (see Participants). The study information page informed participants that they could take part in the online version of the study or request a paper copy of the questionnaires. Two participants 
requested paper copies, which were posted out to them with stamped addressed return envelopes. The questionnaire battery took approximately 30 minutes to complete. Ethical approval for the study was gained from the School of Psychology, University of Leeds University. All participants gave informed consent to participate in the study.

\section{Statistical analysis}

Differences in group scores on the measures of anxiety, stress, depression, dissociation and déjà vu frequency were analysed using independent samples t-tests. The relationship between déjà vu frequency questions, DES scores, and age were analysed using Pearson's correlations. Questions about the triggers of déjà vu, duration of episodes, distress levels and emotionality were analysed using $X^{2}$ tests.

\section{Results}

The main aim of this study was to ascertain whether people who reported a clinical diagnosis of anxiety had a higher level of déjà vu than a comparison Control Group. We thus first concentrate on comparisons between the groups on our déjà vu measures and questions, before examining individual differences in the experience of déjà vu, and factors related to déjà vu experience.

\section{Frequency of déjà vu}

The Anxiety Group reported a higher frequency of déjà vu experiences over the past month $(\mathrm{M}=2.94, \mathrm{SD}=2.70)$ compared to controls $\left(\mathrm{M}=2.30, \mathrm{SD}=1.86 ; \mathrm{t}_{256.66}=\right.$ $2.51, p=0.01, d=0.28$; Figure 2 ). The Anxiety Group also reported a numerically higher frequency of déjà vu experiences over the past year $(\mathrm{M}=7.30, \mathrm{SD}=4.98)$ compared to controls $\left(\mathrm{M}=6.43, \mathrm{SD}=5.07 ; \mathrm{t}_{349}=1.61, p=0.11, d=0.17\right.$; Figure 3$)$, although this difference was not significant. 
Participants were also asked as to whether they perceived their déjà vu experiences to be more frequent than others their age. Overall, 29\% of the Anxiety Group, compared to $17 \%$ of the Control Group, felt that they experienced déjà vu more frequently than other people their age (Table 2).

[Table 2 here]

\section{Duration of déjà vu}

Participants were asked to provide a text description of the typical duration of their déjà vu experiences. These descriptions were coded into seconds, minutes and hours to allow for comparison between groups. The pattern of responses did not differ significantly between the two groups $\left(X^{2}[1, \mathrm{~N}=227]=2.18, \mathrm{p}=0.34\right)$ (Table 3$)$.

[Table 3 here]

\section{Subjective reports of anxiety and déjà vu}

\section{Triggers of déjà vu}

Participants were asked about the perceived circumstantial triggers for their déjà vu and were asked to select as many as they felt related to them. Possible responses were: locations and people (familiar and unfamiliar), conversations, recreational drugs, stress, tiredness and anxiety. Anxiety was the only trigger significantly more frequently reported by the Anxiety Group than the Control Group (Table 4).

[Table 4 here] 


\section{Distress levels}

The Anxiety Group reported déjà vu as causing them undue distress compared to controls $\left(X^{2}[1, \mathrm{~N}=331]=6.46, \mathrm{p}=0.01\right)$ suggesting that experiencing déjà vu affects this group in a different manner compared to healthy controls (Table 5).

\section{[Table 5 here]}

Déjà vu during periods of high anxiety or stress

Participants were asked about their déjà vu experiences during periods of both minimal and high anxiety/stress. The Anxiety Group reported their déjà vu experiences as significantly more frequent $(\mathrm{M}=2.44, \mathrm{SD}=1.04)$ than controls $(\mathrm{M}=2.09 ; \mathrm{SD}=$ $\left.0.87 ; \mathrm{t}_{285.96}=-3.39, \mathrm{p}=0.001\right)$ during periods of high anxiety/stress. They also reported that these déjà vu experiences as significantly more intense $(M=3.00, S D=1.19)$ than controls $\left(\mathrm{M}=2.45, \mathrm{SD}=1.14 ; \mathrm{t}_{340}=4.28, \mathrm{p}=0.00002\right)$. There were no group differences when asked identical questions regarding periods of minimal anxiety/stress.

\section{Emotionality of déjà vu experiences}

The Anxiety Group reported their déjà vu experiences as containing emotional content more frequently than controls $\left[X^{2}(1, \mathrm{~N}=332)=8.76, \mathrm{p}=0.003\right]$. They also rated this content as significantly more emotional $(\mathrm{M}=2.54, \mathrm{SD}=1.44)$ than controls $\left(\mathrm{M}=2.00, \mathrm{SD}=1.39 ; \mathrm{t}_{233.63}=3.38, \mathrm{p}=0.001\right)$. Participants also had the opportunity to provide some qualitative information regarding the nature of their déjà vu and the emotions they experienced. The Anxiety Group described a range of accompanying emotions, including anxiety, stress and confusion (e.g. 'I become very frightened and as if I am stuck in the room in which I am experiencing it') but also more positive emotions such as curiosity, excitement and nostalgia (e.g. '..it makes me feel like I'm special because I'm experiencing something extraordinary', and sometimes both at once 
(e.g. 'typically very nostalgic emotions, but paired with confusion or detached distress/panic due to the fact that I cannot remember the circumstances of first experiencing the emotion'). Control Group participants reported a similar range of positive and negative emotions, but in addition to this several participants in this group did not feel that any emotion was associated with their déjà experiences e.g. 'usually (a) very neutral feeling'.

\section{Correlational Analyses}

Thus far, our data points to our Anxiety Group having more frequent déjà vu (especially during periods of high anxiety), and déjà vu which is triggered by anxiety and is perceived as being more distressing. However, within each group there are differences in how frequently the groups report having déjà vu (Figures 2 and 3). To examine the characteristics of anxiety triggered déjà vu and anxiety and déjà vu more generally we carried out correlational analyses. We carried out a series of correlations separately in each group (Tables 6 and 7).

The uncorrected correlations (Tables 6 and 7) are mostly significant, given our large sample sizes. Here we will focus on correlations within the Anxiety Group and consider $r$ values of .3 and above as medium size effects (Cohen, 1988) and therefore of interest. Firstly, our measures of déjà vu are internally consistent - with a correlation $(\mathrm{r}=.77)$ between the questions about monthly and yearly frequency. The self-rated frequency of déjà vu in the past month correlates with the SAS measure of anxiety $(\mathrm{r}=.30)$, and although significant correlations are found for all the other anxiety and depression measures (except the subjective anxiety measure), these are not of a comparable effect size. Thus, we are fairly confident in saying that amongst our anxious sample, it is those who experience higher levels of anxiety who have higher levels of déjà vu. This pattern is repeated in the question about frequency of déjà vu in 
the past year, with the SAS giving the highest correlation coefficient $(\mathrm{r}=.36)$. Perhaps of most interest, within the Anxiety Group, it appears that those with highest levels of déjà vu are those with higher reports of dissociative experiences (rs of .27 and .38 for monthly and yearly frequency respectively). The DES itself correlates with most of the anxiety measures with high $\mathrm{r}$ values. Within the Control Group, a similar pattern is found, with strong correlations between many of the tasks. Again, SAS seems to be best correlated with reports of déjà vu frequency, and although not quite reaching a medium effect size, there is a correlation between the DES and the monthly and yearly ratings of déjà vu frequency (rs .27 and .25 respectively). In summary, we have been deliberately cautious in describing the patterns of correlations in our data, but it appears that déjà vu experiences are associated with higher scores on measures of anxiety and dissociative experiences.

[Table 6 here]

[Table 7 here]

\section{Discussion}

We found that participants with clinical anxiety reported a higher frequency of déjà vu episodes than a group of healthy age-matched controls, although there were no group differences in the duration of these episodes. The Anxiety Group also reported their déjà vu experiences as being more frequent and of a higher intensity during periods of high anxiety than controls. When asked to identify circumstances or triggers they associated with the onset of déjà vu, these were broadly similar across the two groups, but the Anxiety Group were significantly more likely than controls to report anxiety as a trigger. They were also significantly more likely to report these episodes contained emotional content. When asked about their feelings towards déjà vu, the 
Anxiety Group reported finding these as significantly more distressing than controls. Perhaps unsurprisingly then, measures of anxiety correlated with the frequency of déjà vu experience.

It has long been established that déjà vu involves memory-related circuitry (e.g. see Hughlings-Jackson, 1881; Penfield \& Perot, 1963; Halgren, Walter, Cherlow \& Crandall, 1978) but research has also long-since implicated emotional circuitry in déjà vu (e.g. Gloor, Oliver \& Quesney, 1982). Structural differences in memory and emotion-related circuitry are apparent when comparing healthy individuals who experience déjà vu and individuals with TLE and déjà vu (Labate et al, 2015), and indeed research comparing the phenomenology of déjà vu in these two groups remains somewhat inconclusive as to the extent to which they are the same (Warren-Gash \& Zeman, 2001; Moulin, 2014; Perucca et al, 2017). Perhaps then, the phenomenological differences we report between our controls and the Anxiety Group also reflect underlying structural differences in memory and/or emotion-related circuitry. Whilst this is beyond the scope of this paper, it would be of interest to determine whether there are any structural markers which differentiate these two groups.

Oscillatory activity may also play a role in the group differences we observe here. Stimulation of the rhinal cortex can induce déjà vu episodes (e.g. Bancaud et al, 1994; Barbeau et al, 2005; Bartolomei et al, 2012) which are accompanied by theta synchronisation across MTL structures (Barbeau et al, 1994; Bartolomei et al, 2012). Theta oscillations are known to play a role in both memory-related processes (e.g. Mormann et al, 2008; Osipova et al, 2006; Sederberg, Kahana, Howard, Donner \& Madsden, 2003), but they also play a role in anxiety-related processing (e.g. Cornwell, Arkin, Overstreet, Carver, \& Grillon, 2012; Khemka, Barnes, Dolan \& Bach, 2017). In addition, O'Connor et al (2010) speculatively proposed theta oscillations could play a 
role in déjà vécu, a clinical manifestation of déjà vu. If theta is involved in the generation of déjà vu perhaps the differences we observed between the Anxiety Group and controls reflect underlying anxiety-related differences in oscillatory activity.

We found that whilst there were differences in the frequency of déjà vu episodes, and the perceived distress they caused, there was no difference in duration of déjà vu when comparing the two groups. However, one of the key limitations in déjà vu research is that it is largely retrospective in nature, which is problematic when aiming to collect accurate data about these subjective experiences. We attempted to address this issue by encouraging participants who completed the study discussed here to complete a brief follow-up immediately after their next déjà vu episode. This follow-up required participants to complete the Impact of Event Scale (Horowitz, Wilner \& Alvarez, 1979), typically used to assess stress reactions for traumatic events. Low numbers of respondents precluded us from engaging in a detailed analysis, but we did find that the Anxiety Group scored significantly higher than controls on the intrusion subscale, indicating that these participants experienced intrusive thoughts about their déjà vu following that specific episode. This is interesting when considering that in this paper we report Anxiety Group found déjà vu to be significantly more distressing than the Control Group did. It would therefore be useful to further develop this follow-up approach, asking some more detailed questions regarding those recently-experienced déjà vu experiences. It would also be interesting to gather detailed data on vividness, as there is some retrospective data indicating that this can differ between clinical groups (Harper \& Roth, 1962). Another issue we believe to be of interest is determining the relationship between déjà vu intensity and frequency, and whether the nature of this relationship would differ between clinically anxious individuals and controls. 
We have added to the evidence base about clinical anxiety and déjà vu. It is commonly asserted there is a relationship between déjà vu and stress (e.g. Brown, 2003), and more recently research has indicated that there may be differences in the frequency and intensity of déjà vu experienced by people with clinical anxiety. We therefore believe that this paper adds important quantitative support, which has been previously lacking, to the existence of such relationships. This paper therefore contributes not only to the pre-existing body of déjà vu research, but also makes a clinically-relevant contribution. Towards the end of this study we asked participants whether they have ever discussed their déjà vu experiences when with a medical professional. In a few instances, participants reported that they had, and that they were told anxiety and déjà vu are associated (e.g. 'yes, (I) was told it was common for people with anxiety'). In presenting novel findings which confirm the existence of such a relationship, our findings therefore strengthen the validity of such discussions between patients and clinicians.

In summary, we report the main findings from the first large-scale study profiling déjà vu in anxiety. It would appear that déjà vu is more frequently experienced by people with clinical anxiety, and that déjà vu experiences cause significantly more distress than they do in healthy controls. Understanding these group differences may be of use to clinicians when considering the potential impact of déjà vu symptoms upon the psychological wellbeing of individuals with anxiety disorders.

Acknowledgements: The authors are grateful to Dave Horton for helping set up the online questionnaire.

\section{References:}

Adachi, N., Akanu, N., Adachi, T., Takekawa, Y., Adachi, Y., Ito, M., \& Ikeda, H. (2008). Déjà vu experiences are rarely associated with pathological dissociation. 
Journal of Nervous and Mental Disorders, 196(5), 417-419. doi:

10.1097/NMD.0b013e31816ff36d

Adachi, T., Adachi, N., Takekawa, Y., Akanuma, N., Ito, M., Matsubara, R., Ikeda, H. et al. (2006). Déjà vu experiences in patients with schizophrenia. Comprehensive Psychiatry, 47(5), 389-393. doi:10.1016/j.comppsych.2005.12.003

Bancaud, J., Brunet-Bourgin, F., Chauvel, P. \& Halgren, E. (1994). Anatomical origin of déjà vu and vivid 'memories' in human temporal lobe epilepsy. Brain, 117(1), 71-90. doi: 10.1093/brain/117.1.71

Barbeau, E., Wendling, F., Régis, J., Duncan, R., Poncet, M., Chauvel, P., \& Bartolomei, F. (2005). Recollection of vivid memories after perirhinal region stimulations: synchronization in the theta range of spatially distributed brain areas. Neuropsychologia, 43(9), 1329-1337. doi:

10.1016/j.neuropsychologia.2004.11.025

Bartolomei, F., Barbeau, E. J., Nguyen, T., McGonigal, A., Régis, J., Chauvel, P., \& Wendling, F. (2012). Rhinal-hippocampal interactions during déjà vu. Clinical Neurophysiology, 123(3), 489-495. doi: 10.1016/j.clinph.2011.08.012

Bech, P., Rasmussen, N-A., Olsen, L.R., Noerholm, V., \& Abildgaard, W. (2001). The sensitivity and specificity of a Major Depression Inventory, using the Present State Examination as the index of diagnostic validity. Journal of Affective Disorders, 66, 159164. doi:10.1016/S0165-0327(00)00309-8

Bernstein, E.M., \& Putnam, F.W. (1993). An update on the Dissociative Experiences Scale. Dissociation: Progress in the Dissociative Disorders, 6, 16-27.

Brázdil, M., Mareček, R., Fojtíková, D., Mikl, M., Kuba, R., Krupa, P., \& Rektor, I. (2009). Correlation study of optimized voxel-based morphometry and 1H MRS in patients with mesial temporal lobe epilepsy and hippocampal sclerosis. Human brain mapping, 30(4), 1226-1235. doi: 10.1002/hbm.20589

Brázdil, M., Mareček, R., Urbanek, T., Kašparek, T., Mikl, M., Rektor, I., \& Zeman, A. (2012). Unveiling the mystery of déjà vu: the structural anatomy of déjà vu. cortex, 48(9), 1240-1243. doi: 10.1016/j.cortex.2012.03.004 
Brown, A. S. (2003). A review of the déjà vu experience. Psychological Bulletin, 129(3), 394-413. doi: 10.1037/0033-2909.129.3.394

Cornwell, B. R., Arkin, N., Overstreet, C., Carver, F. W., \& Grillon, C. (2012). Distinct contributions of human hippocampal theta to spatial cognition and anxiety. Hippocampus, 22(9), 1848-1859. doi: 10.1002/hipo.22019

Eichenbaum, H., Yonelinas, A. P., \& Ranganath, C. (2007). The medial temporal lobe and recognition memory. Annu. Rev. Neurosci., 30, 123-152. doi: 10.1146/annurev.neuro.30.051606.094328

Fontani, G., Farbollini, F., \& Carli, G. (1984). Hippocampal electrical activity and behavior in the presence of novel environmental stimuli in rabbits. Behavioural brain research, 13(3), 231-240. doi: 10.1016/0166-4328(84)90165-7

Fontani, G., \& Vegni, V. (1990). Hippocampal electrical activity during social interactions in rabbits living in a seminatural environment. Physiology \& behavior, 47(1), 175-183. doi: 10.1016/0031-9384(90)90058-C

Fontani, G., \& Vegni, V. (1990). Hipppcampal Electrical Activity and Behaviour in Rabbits during Adaptation to a Novel Semi-Natural Environment. European Journal of Neuroscience, 2(3), 203-210. doi: 10.1111/j.1460-9568.1990.tb00413.x

Gloor, P., Olivier, A., Quesney, L. F., Andermann, F., \& Horowitz, S. (1982). The role of the limbic system in experiential phenomena of temporal lobe epilepsy. Annals of neurology, 12(2), 129-144.

Gordon, J. A., Lacefield, C. O., Kentros, C. G., \& Hen, R. (2005). State-dependent alterations in hippocampal oscillations in serotonin 1A receptor-deficient mice. Journal of Neuroscience, 25(28), 6509-6519. DOI: doi: 10.1523/JNEUROSCI.1211-05.2005

Gray, J.A. The Neuropsychology of Anxiety. New York: Oxford University Press.

Gray, J.A. \& McNaughton, N. (2000). The Neuropsychology of Anxiety (2 ${ }^{\text {nd }}$ Edition). Oxford: Oxford University Press. 
Halgren, E., Walter, R.D., Cherlow, D.G. \& Crandall, P.H. (1978). Mental phenomena evoked by electrical stimulation of the human hippocampal formation and amygdala. Brain, 101(1), 83-115.

Harper, M., \& Roth, M. (1962). Temporal lobe epilepsy and the phobic anxietydepersonalisation syndrome. Part 1. A Comparative Study. Comprehensive Psychiatry, 3, 129-151. doi: 10.1016/S0010-440X(62)80009-1

Horowitz, M., Wilner, N., Alvarez, W. (1979). Impact of event scale: a measure of subjective stress. Psychosomatic Medicine, 41, 209-18.

Hughlings-Jackson, J. (1881). On temporary paralysis after epileptiform and epileptic seizures; a continuation to the study of dissolution of the nervous system. Brain, 3(4), 433-451.

Illman, N.A., Butler, C.R., Souchay, C., \& Moulin, C.J.A. (2012). Deja experiences in temporal lobe epilepsy. Epilepsy Research and Treatment, 2012, 1-15. doi:

$10.1155 / 2012 / 539567$

Khemka, S., Barnes, G., Dolan, R. J., \& Bach, D. R. (2017). Dissecting the function of hippocampal oscillations in a human anxiety model. Journal of Neuroscience, 1834-16. doi: 10.1523/JNEUROSCI.1834-16.2017

Labate, A., Cerasa, A., Mumoli, L., Ferlazzo, E., Aguglia, U., Quattrone, A., \& Gambardella, A. (2015). Neuro-anatomical differences among epileptic and nonepileptic déjà-vu. cortex, 64, 1-7. doi: 10.1016/j.cortex.2014.09.020

Lovibond, S.H., \& Lovibond, P.F. (1995). Manual for the depression anxiety stress scales. Sydney: Psychology Foundation.

Mormann, F., Osterhage, H., Andrzejak, R. G., Weber, B., Fernández, G., Fell, J., ... \& Lehnertz, K. (2008). Independent delta/theta rhythms in the human hippocampus and entorhinal cortex. Frontiers in human neuroscience, 2, 3. doi:

10.3389/neuro.09.003.2008 
Moulin, C.J.A. (2014). The strange sensation of déjà vu: not so strange in temporal lobe epilepsy. Journal of Neurology, Neurosurgery \& Psychiatry, 85, 132. doi:

10.1136/jnnp-2012-303876

Pail, M., Brázdil, M., Mareček, R., \& Mikl, M. (2010). An optimized voxel-based morphometric study of grey matter changes in patients with left-sided and right-sided mesial temporal lobe epilepsy and hippocampal sclerosis (MTLE/HS). Epilepsia, 51(4), 511-518. doi: 10.1111/j.1528-1167.2009.02324.x

Penfield, W., \& Perot, P. (1963). The brain's record of auditory and visual experience: a final summary and discussion. Brain, 86(4), 595-696.

O'Connor, A. R., Lever, C., \& Moulin, C. J. (2010). Novel insights into false recollection: a model of déjà vécu. Cognitive Neuropsychiatry, 15(1-3), 118-144. doi: $10.1080 / 13546800903113071$

Osipova, D., Takashima, A., Oostenveld, R., Fernández, G., Maris, E., \& Jensen, O. (2006). Theta and gamma oscillations predict encoding and retrieval of declarative memory. Journal of neuroscience, 26(28), 7523-7531. doi: 10.1523/JNEUROSCI.194806.2006

Perucca, P., Crompton, D. E., Bellows, S. T., McIntosh, A. M., Kalincik, T., Newton, M. R., ... \& Tan, K. M. (2017). Familial mesial temporal lobe epilepsy and the borderland of déjà vu. Annals of neurology, 82(2), 166-176. doi: 10.1002/ana.24984

Roth, M. (1959). The phobic anxiety-depersonalization syndrome. Proceedings of the Royal Society of Medicine, 52, 587-595.

Sederberg, P. B., Kahana, M. J., Howard, M. W., Donner, E. J., \& Madsen, J. R. (2003). Theta and gamma oscillations during encoding predict subsequent recall. Journal of Neuroscience, 23(34), 10809-10814. doi: 10.1523/JNEUROSCI.23-34-10809.2003

Shaw, D. J., Mareček, R., \& Brázdil, M. (2016). Structural covariance mapping delineates medial and medio-lateral temporal networks in déjà vu. Brain imaging and behavior, 10(4), 1068-1079. doi: 10.1007/s11682-015-9471-8 
Silberman, E.K., Post, R.M., Nurnberger, J., Theodore, W., Boulenger, J-P. (1985).

Sensory, cognitive and affective phenomena in affective illness: comparison with partial epilepsy. British Journal of Psychiatry, 146, 81-89. doi: 10.1192/bjp.146.1.81

Sno, H. N., Schalken, H. F., de Jonghe, F., \& Koeter, M. W. (1994). The Inventory for

Déjà vu Experiences Assessment: Development, utility, reliability, and validity. Journal of Nervous and Mental Disease, 182(1), 27-33. doi: 10.1097/00005053-19940100000006

Squire, L. R., Wixted, J. T., \& Clark, R. E. (2007). Recognition memory and the medial temporal lobe: a new perspective. Nature Reviews Neuroscience, 8(11), 872. doi: $10.1038 / \mathrm{nrn} 2154$

Urquhart, J. A., Sivakumaran, M. H., Macfarlane, J. A., \& O’Connor, A. R. (2018). fMRI evidence supporting the role of memory conflict in the déjà vu experience. Memory, 1-12. doi: 10.1080/09658211.2018.1524496

Warren-Gash, C., \& Zeman, A. (2014). Is there anything distinctive about epileptic déjà vu? Journal of Neurology, Neurosurgery \& Psychiatry, 85, 143-147. doi: 10.1136/jnnp2012-303520

Warren-Gash, C., \& Zeman, A. (2003). Déjà vu. Practical Neurology, 3, 106-9. doi: 10.1046/j.1474-7766.2003.11136.x

Wells, C.E., Moulin, C.J.A., Ethridge, P., Illman, N.A., Davies, E., Zeman, A. (2014). Persistent psychogenic déjà vu: a case report. Journal of Medical Case Reports, 8, 414417. doi: 10.1186/1752-1947-8-414

Wells, C. E., Amos, D. P., Jeewajee, A., Douchamps, V., Rodgers, J., O'Keefe, J., ... \& Lever, C. (2013). Novelty and anxiolytic drugs dissociate two components of hippocampal theta in behaving rats. Journal of Neuroscience, 33(20), 8650-8667. DOI: doi: 10.1523/JNEUROSCI.5040-12.2013

Zung, W.W.K. (1971). A rating instrument for anxiety disorder. Psychosomatics, 12, 371-379. doi: 10.1016/S0033-3182(71)71479-0 
Table 1. Anxiety and Control Group descriptives and comparisons on measures of depression, anxiety, stress and dissociative experiences

\begin{tabular}{|c|c|c|c|c|c|c|}
\hline \multirow[t]{3}{*}{ Measure } & $\begin{array}{l}\text { Anxiety } \\
\text { group }\end{array}$ & $\begin{array}{l}\text { Control } \\
\text { group }\end{array}$ & \multirow[t]{3}{*}{$t$} & \multirow[t]{3}{*}{$d f$} & \multirow[t]{3}{*}{$P$} & \multirow[t]{3}{*}{$\begin{array}{l}\text { Cohen's } \\
d\end{array}$} \\
\hline & $(\mathrm{n}=153)$ & $(\mathrm{n}=199)$ & & & & \\
\hline & Mean (SD) & $\begin{array}{l}\text { Mean } \\
(\mathrm{SD})\end{array}$ & & & & \\
\hline DES & $\begin{array}{l}27.57 \\
(19.47)\end{array}$ & $\begin{array}{l}19.90 \\
(15.44)\end{array}$ & 4.01 & 283.53 & $\mathrm{p}<.001$ & 0.40 \\
\hline DASS & 18.50 & 11.05 & 6.74 & 303.17 & $\mathrm{p}<.001$ & 0.55 \\
\hline Anxiety & $(10.85)$ & $(9.05)$ & & & & \\
\hline DASS Stress & $\begin{array}{l}23.44 \\
(10.62)\end{array}$ & $\begin{array}{l}15.72 \\
(10.12)\end{array}$ & 6.94 & 350 & $\mathrm{p}<.001$ & 0.79 \\
\hline DASS & 21.29 & 12.47 & 6.74 & 306.49 & $\mathrm{p}<.001$ & 0.78 \\
\hline Depression & (12.76) & (11.36) & & & & \\
\hline SAS & $\begin{array}{l}60.37 \\
(14.01)\end{array}$ & $\begin{array}{l}49.23 \\
(12.40)\end{array}$ & 7.78 & 305.28 & $\mathrm{p}<.001$ & 0.89 \\
\hline MDI & $\begin{array}{l}26.59 \\
(12.54)\end{array}$ & $\begin{array}{l}17.45 \\
(11.78)\end{array}$ & 7.18 & 350 & $\mathrm{p}<.001$ & 0.74 \\
\hline $\begin{array}{l}\text { Subjective } \\
\text { anxiety }\end{array}$ & $4.53(0.63)$ & $3.39(1.06)$ & 12.53 & 339.53 & $\mathrm{p}<.001$ & 0.13 \\
\hline
\end{tabular}

Note: DES is Dissociative Experiences Scale, DASS is Depression Anxiety Stress Scales, SAS is Zung Self-Rating Anxiety Scale, MDI is Major Depression Inventory. T $=\mathrm{t}$ value for independent $\mathrm{t}$-test, $\mathrm{df}=$ degrees of freedom, $\mathrm{p}=$ significance value, Cohen's $d=$ effect size. T-test values ( $t$, $d f \&$ p) reported according to Levene's test. Bonferroni adjusted alpha levels of $\mathrm{p}<.007$. 
Table 2. Participants' beliefs about the frequency of their déjà vu experiences

\begin{tabular}{lll}
\hline $\begin{array}{l}\text { More than } \\
\text { others }\end{array}$ & Anxiety Group & Control Group \\
same age & & \\
\hline Yes & $28.5 \%$ & $17.2 \%$ \\
No & $23.2 \%$ & $31.3 \%$ \\
Unsure & $48.3 \%$ & $51.6 \%$ \\
\hline
\end{tabular}


Table 3. Duration of déjà vu episodes in the Anxiety and Control Groups

\begin{tabular}{lll}
$\begin{array}{l}\text { Duration } \\
\text { of Déjà }\end{array}$ & Anxiety Group & Control Group \\
$\mathrm{Vu}$ & & \\
\hline Seconds & $42.3 \%$ & $47.1 \%$ \\
Minutes & $52.9 \%$ & $51.3 \%$ \\
Hours & $4.8 \%$ & $1.7 \%$ \\
\hline
\end{tabular}


Table 4. Percentage of participants reporting each type of trigger preceding their déjà vu

\begin{tabular}{|c|c|c|c|c|c|}
\hline \multirow[t]{3}{*}{ Trigger } & \multicolumn{5}{|c|}{$\%$ experienced trigger } \\
\hline & Anxiety & Control & $X^{2}$ & $d f$ & $P$ \\
\hline & Group & Group & & & \\
\hline Conversation & 55.60 & 55.80 & 0.05 & 1 & 0.82 \\
\hline Familiar location & 48.40 & 49.70 & 0.07 & 1 & 0.80 \\
\hline Unfamiliar location & 26.80 & 20.10 & 2.19 & 1 & 0.14 \\
\hline Familiar people & 42.50 & 43.20 & 0.02 & 1 & 0.89 \\
\hline Unfamiliar people & 18.30 & 18.60 & 0.01 & 1 & 0.94 \\
\hline Anxiety & 24.80 & 10.60 & 12.65 & 1 & 0.0004 \\
\hline Stress & 20.90 & 14.10 & 2.87 & 1 & 0.09 \\
\hline Recreational drugs & 3.90 & 5.00 & 0.24 & 1 & 0.62 \\
\hline $\begin{array}{l}\text { Tiredness/sleep } \\
\text { deprivation }\end{array}$ & 24.20 & 26.60 & 0.27 & 1 & 0.60 \\
\hline $\begin{array}{l}\text { No particular } \\
\text { triggers }\end{array}$ & 27.50 & 25.6 & 0.15 & 1 & 0.70 \\
\hline
\end{tabular}

Note: $\mathrm{X}^{2}=$ Chi Squared, $\mathrm{df}=$ degrees of freedom, $\mathrm{p}=$ significance value, Bonferroni adjusted alpha levels of $\mathrm{p}<005$. 
Table 5. Presence of déjà vu-related distress reported by the Anxiety and Control Groups

\begin{tabular}{lll}
\hline $\begin{array}{l}\text { Undue } \\
\text { distress }\end{array}$ & Anxiety Group & Control Group \\
caused by & & \\
déjà vu? & & \\
\hline Yes & $17.24 \%$ & $8.06 \%$ \\
No & $82.76 \%$ & $91.94 \%$ \\
\hline
\end{tabular}


Table 6. Pearson's correlations for the Anxiety Group: déjà vu frequency, age, measures of anxiety, stress, depression and dissociation

\begin{tabular}{|c|c|c|c|c|c|c|c|c|c|c|}
\hline & Age & $\begin{array}{l}\text { Déjà vu } \\
\text { frequency } \\
\text { (times past } \\
\text { month) }\end{array}$ & $\begin{array}{l}\text { Déjà vu } \\
\text { frequency } \\
\text { (times past } \\
\text { year) }\end{array}$ & DES & $\begin{array}{l}\text { DASS } \\
\text { Anxiety }\end{array}$ & $\begin{array}{l}\text { DASS } \\
\text { Stress }\end{array}$ & $\begin{array}{l}\text { DASS } \\
\text { Depression }\end{array}$ & SAS & MDI & $\begin{array}{l}\text { Subjective } \\
\text { anxiety }\end{array}$ \\
\hline Age & - & $-.20^{*}$ & $-.17^{*}$ & -.01 & $-.16^{*}$ & -.11 & -.03 & $.16^{*}$ & -.02 & -.13 \\
\hline $\begin{array}{l}\text { Déjà vu frequency } \\
\text { (times past month) }\end{array}$ & & - & $0.77^{* * *}$ & $0.27 * *$ & $.21^{* *}$ & $.21 *$ & $.23^{* *}$ & $.30^{* *}$ & $.26 * *$ & -.06 \\
\hline $\begin{array}{l}\text { Déjà vu frequency } \\
\text { (times past year) }\end{array}$ & & & - & $0.38 * * *$ & $.26^{* *}$ & $.25 * *$ & $.18^{*}$ & $.36 * * *$ & $.25^{* *}$ & .01 \\
\hline DES & & & & - & $.47 * * *$ & $.43 * * *$ & $.38 * * *$ & $.54 * * *$ & $.47 * * *$ & $.18^{*}$ \\
\hline DASS Anxiety & & & & & - & $.69 * * *$ & $.58 * * *$ & $.81 * * *$ & $.60 * * *$ & $.34 * * *$ \\
\hline DASS Stress & & & & & & - & $.62 * * *$ & $.74 * * *$ & $.67 * * *$ & $.36^{* * *}$ \\
\hline DASS Depression & & & & & & & - & $.61 * * *$ & $.81 * * *$ & $.17^{*}$ \\
\hline SAS & & & & & & & & - & $.73 * * *$ & $.32 * * *$ \\
\hline MDI & & & & & & & & & - & $.24 * *$ \\
\hline Subjective anxiety & & & & & & & & & & - \\
\hline
\end{tabular}

Note: $\mathrm{n}=153,{ }^{*} \mathrm{p}<0.05,{ }^{* *} \mathrm{p}<0.01, * * * \mathrm{p}<0.001$. DES is Dissociative Experiences Scale, DASS is Depression Anxiety Stress Scales, SAS is Zung Self-Rating Anxiety Scale, MDI is Major Depression Inventory. 
Table 7. Pearson's correlations for the Control Group: déjà vu frequency, age, measures of anxiety, stress, depression and dissociation

\begin{tabular}{|c|c|c|c|c|c|c|c|c|c|c|}
\hline & Age & $\begin{array}{l}\text { Déjà vu } \\
\text { frequency } \\
\text { (times past } \\
\text { month) }\end{array}$ & $\begin{array}{l}\text { Déjà vu } \\
\text { frequency } \\
\text { (times past } \\
\text { year) }\end{array}$ & DES & $\begin{array}{l}\text { DASS } \\
\text { Anxiety }\end{array}$ & $\begin{array}{l}\text { DASS } \\
\text { Stress }\end{array}$ & $\begin{array}{l}\text { DASS } \\
\text { Depression }\end{array}$ & SAS & MDI & $\begin{array}{l}\text { Subjective } \\
\text { anxiety }\end{array}$ \\
\hline Age & - & $-.25^{* * *}$ & $-.21 * *$ & $-.18^{* *}$ & $-.21 * *$ & -.09 & $-.15^{*}$ & $-.21 * *$ & $-.15^{*}$ & -.07 \\
\hline $\begin{array}{l}\text { Déjà vu frequency } \\
\text { (times past month) }\end{array}$ & & - & $.65^{* * *}$ & $.27 * * *$ & $.27 * * *$ & $.23 * *$ & $.23 * *$ & $.33^{* * * *}$ & $.25 * * *$ & .07 \\
\hline $\begin{array}{l}\text { Déjà vu frequency } \\
\text { (times past year) }\end{array}$ & & & - & $.25 * * *$ & $.29 * * *$ & $.21 * *$ & $.24 * *$ & $.24 * *$ & $.20 * *$ & .06 \\
\hline DES & & & & - & $.49 * * *$ & $.45^{* * *}$ & $.43 * * *$ & $.44 * * *$ & $.54 * * *$ & .03 \\
\hline DASS Anxiety & & & & & - & $.76^{* * *}$ & $.62 * * *$ & $.80 * * *$ & $.69 * * *$ & $.37 * * *$ \\
\hline DASS Stress & & & & & & - & $.68 * * *$ & $.75^{* * *}$ & $.72 * * *$ & $.42 * * *$ \\
\hline DASS Depression & & & & & & & - & $.62 * * *$ & $.83 * * *$ & $.35^{* * *}$ \\
\hline SAS & & & & & & & & - & $.76^{* * *}$ & $.47 * * *$ \\
\hline MDI & & & & & & & & & - & $.36^{* * *}$ \\
\hline Subjective anxiety & & & & & & & & & & _ \\
\hline
\end{tabular}

Note: $\mathrm{n}=199,{ }^{*} p<0.05,{ }^{*} p<0.01,{ }^{* * *} p<0.001$. DES is Dissociative Experiences Scale, DASS is Depression Anxiety Stress Scales, SAS is Zung Self-Rating Anxiety Scale, MDI is Major Depression Inventory. 


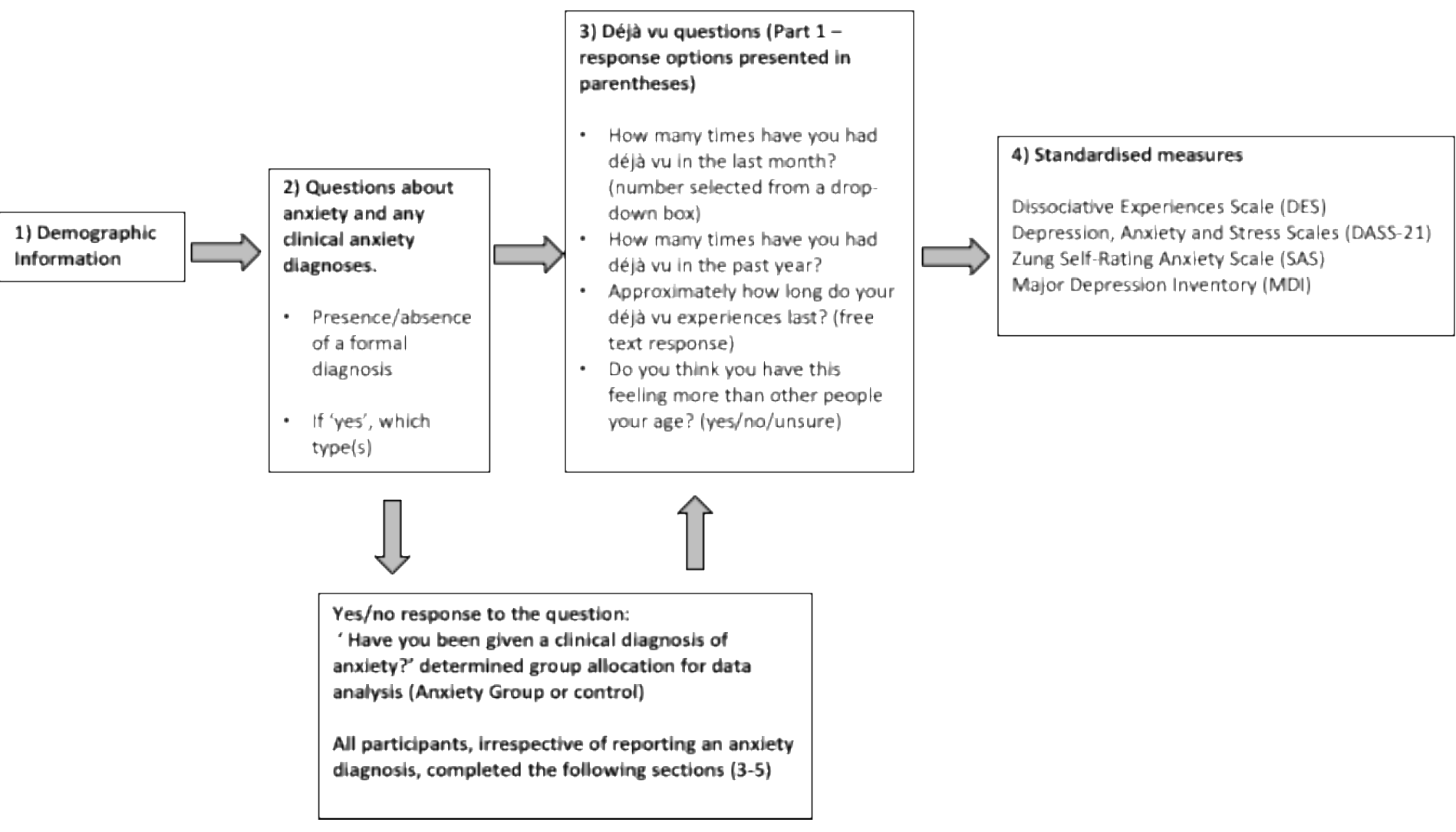

5) Déjà vu questions (Part 2 - response options

presented in parentheses)

Questions about déjà vu in relation to anxiety mor

specifically:

- When you are experiencing a period of minimal anxiety or stress, how frequently do you experience déjà vu?

(daily/weekly/monthly/sometimes/never)

- How intense are these experiences (Likert scale: 1 $=$ not at all intense, 5 = very intense)

- When you are experiencing a period of minimal anxiety or stress, how frequently do you experience déjà vu?

(daily/weekly/monthly/sometimes/never)

- How intense are these experiences (Likert scale: 1 $=$ not at all intense, $5=$ very intense)

Are there any particular triggers for your déjà v experiences? Select as many options as apply to you (familiar location, unfamiliar location,

conversations, familiar people, unfamiliar people, recreational drugs, stress, anxiety, tiredness/sleep deprivation, no particular triggers).

- Do your déjá vu experiences cause you undue distress? (yes/no)

Do your experiences contain any emotiona content? (yes/no)

Figure 1. Structure of the online study \& order in which materials were presented to participants. 


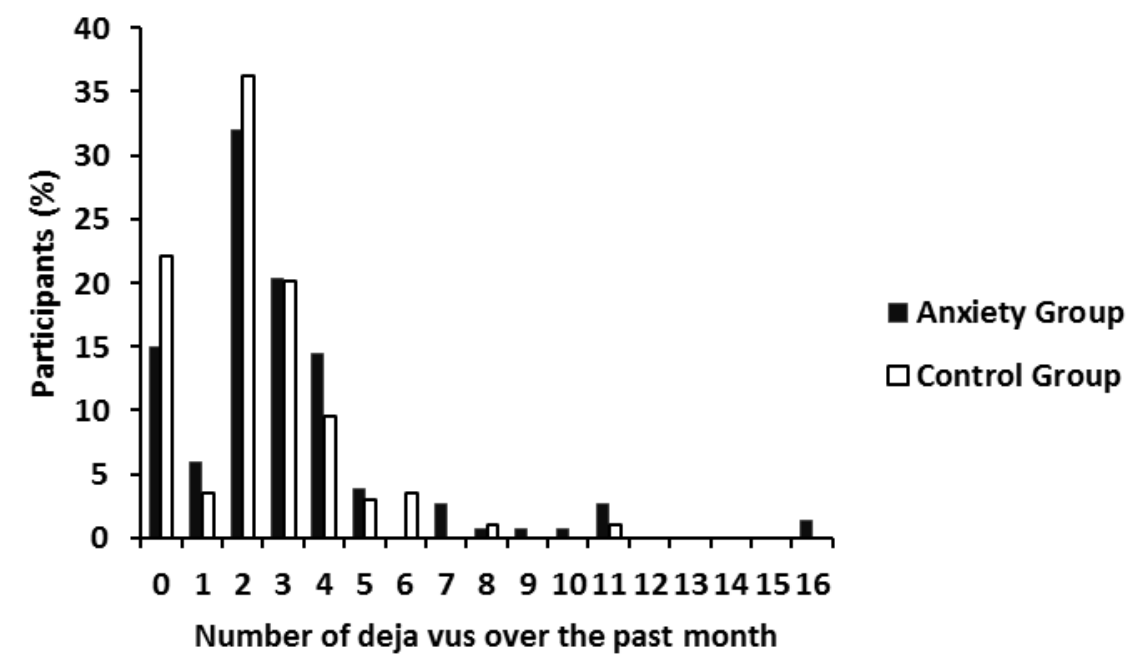

Figure 2. The comparative frequency of déjà vu episodes experienced over the past month by the Anxiety and Control Groups 


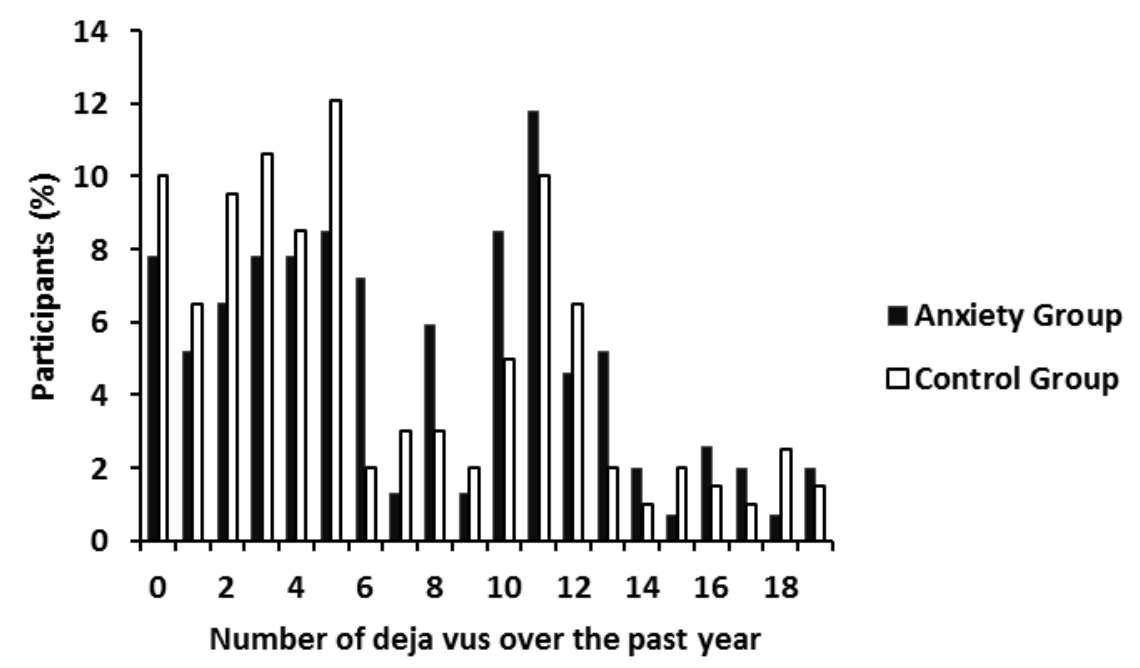

Figure 3. The comparative frequency of déjà vu episodes experienced over the past year by the Anxiety and Control Group 